\title{
IPS OBSERVATIONS OF SHORT-TIME SCALE INTERPLANETARY ACTIVITY
}

\author{
PRADEEP GOTHOSKAR AND A. PRAMESH RAO \\ National Centre for Radio Astrophysics, TIFR, \\ Poona University Campus, Pune 411 007. India
}

\begin{abstract}
We have carried out a program of continuous Interplanetary Scintillation (IPS) monitoring of the interplanetary activity using Ooty Radio Telescope (ORT). From May 1990 to March 1991 , during the $22^{\text {nd }}$ solar maximum, a few radio sources were monitored to provide long stretches of IPS data with a high-time resolution of few minutes. These observations covered 0.3 to $0.8 \mathrm{AU}$ region $\left(12^{\circ}\right.$ to $70^{\circ}$ elongations $)$ around the sun at several heliographic latitudes. During the observation, we detected 33 short-time scale IPS events which had significant variation in the scintillation index and solar wind velocity. These were considered to be due to travelling interplanetary disturbances.

A multi-component model of plasma density enhancement was developed to estimate the geometry and physical properties of these IPS events. Detailed analysis of 20 of these events suggests, 1. fast IPS events were interplanetary signatures of Coronal Mass Ejections (CMEs), 2. the average mass and energy of these events was $\sim 10^{16} \mathrm{gm}$ and $10^{33}$ erg respectively, 3. $80 \%$ of IPS events were associated with X-ray flares on the sun and $50 \%$ were associated with geomagnetic activity at earth. Detailed study of the multicomponent model suggests IPS observations at smaller elongations (hence at higher radio frequencies) are more suited to detect fast-moving interplanetary disturbances such as produced by CMEs.
\end{abstract}

Key words: Solar Wind, Solar Terrestrial Physics, Interplanetray Scintillations

\section{IPS Monitoring Programme}

Interplanetary Scintillation (IPS) is produced when radio waves from a compact radio source are scattered due to the density fluctuations in the solar wind. In the region of weak scattering (where the $r m s$ phase fluctuations are less than $1 \mathrm{rad}$ ), characteristics of scintillation provides information about the average density and velocity of the plasma along the line of sight. Hence, the technique is extensively used to study the properties of the interplanetary medium and interplanetary activity (Gapper et al 1982, Tappin et al 1983). However due to various limitations of telescopes, IPS observations in the past were more sensitive to long duration (hence slow moving) interplanetary disturbances. In order to study interplanetary activity over short-time scales of a few minutes to a few hours, we carried out a programme of continuous IPS monitoring during the $22^{\text {nd }}$ solar maximum. During May 1990 and March 1991, we observed 14 strong IPS sources over elongation of $20^{\circ}$ to $60^{\circ}$ and heliographic latitudes $0^{\circ}$ to $80^{\circ}$. Every day, one IPS source was observed continuously for $\sim 8.5$ hours to provide a long stretch of scintillation data. After calibrating and removing intereference, the data were analysed to provide time series of IPS power spectra, scintillation index and solar wind 
velocity. Initial estimates of the solar wind velocity were obtained using the method of single station velocity estimation (Manoharan \& Ananthakrishnan, 1990).

On 59 days (out of a total of 210 days) we observed significant variation in scintillation index and solar wind velocity within 8 hours. These days were classified as IPS-active days. Of these, 33 days, which showed a systematic variation in index and velocity were selected as IPS-event days. Considering the time scale of index and velocity variation, these events were classified as slow ( $>4$ hours), medium ( $<4$ hours) and fast (few minutes) events. Figure 1 shows two of the fast IPS events observed on July $31^{\text {st }} 1990$ and August $25^{\text {th }} 1990$. Observations showed simultaneuous increase in the scintillation index and velocity within a few minutes. The index and velocity returned to their normal values after the event. The variation in index and velocity, observed during an IPS event, was attributed to an interplanetary disturbance crossing the line of sight. We developed a multi-component model of scattering by interplanetary disturbances to model the 33 IPS events.

\section{Multi-component IPS model}

The medium along the line of sight was divided into two components namely 'event component' and 'normal component'. While normal component represented quiet solar wind with normal velocity and density, event component was due to interplanetary disturbance travelling across the line of sight with different density and velocity. Typically, interplanetary disturbance was considered as a dense high-velocity cloud of plasma travelling with specified geometry. Contribution of interplanetary disturbance to scattering thus varied with time resulting in time series of model power spectra, scintillation index and velocity. The detailed profile of variation of index and velocity was related to the geometry of disturbance, such as ejection angle and size, and physical properties, such as density and velocity. The observed time series of index and velocity was modeled using the multi-component model to obtain reasonable visual match. Figure 1 shows the time series of model index and velocity (bold line) superposed onto observed series of index and velocity (thin line). Of the 33 IPS events, we could model 20 events using the multi-component model and obtained velocity, mass and kinetic energy of the interplanetary disturbances.

\section{Results}

Detailed study of the multi-component model was carried out considering mass ejection at several angles observed at different elongations and radio 

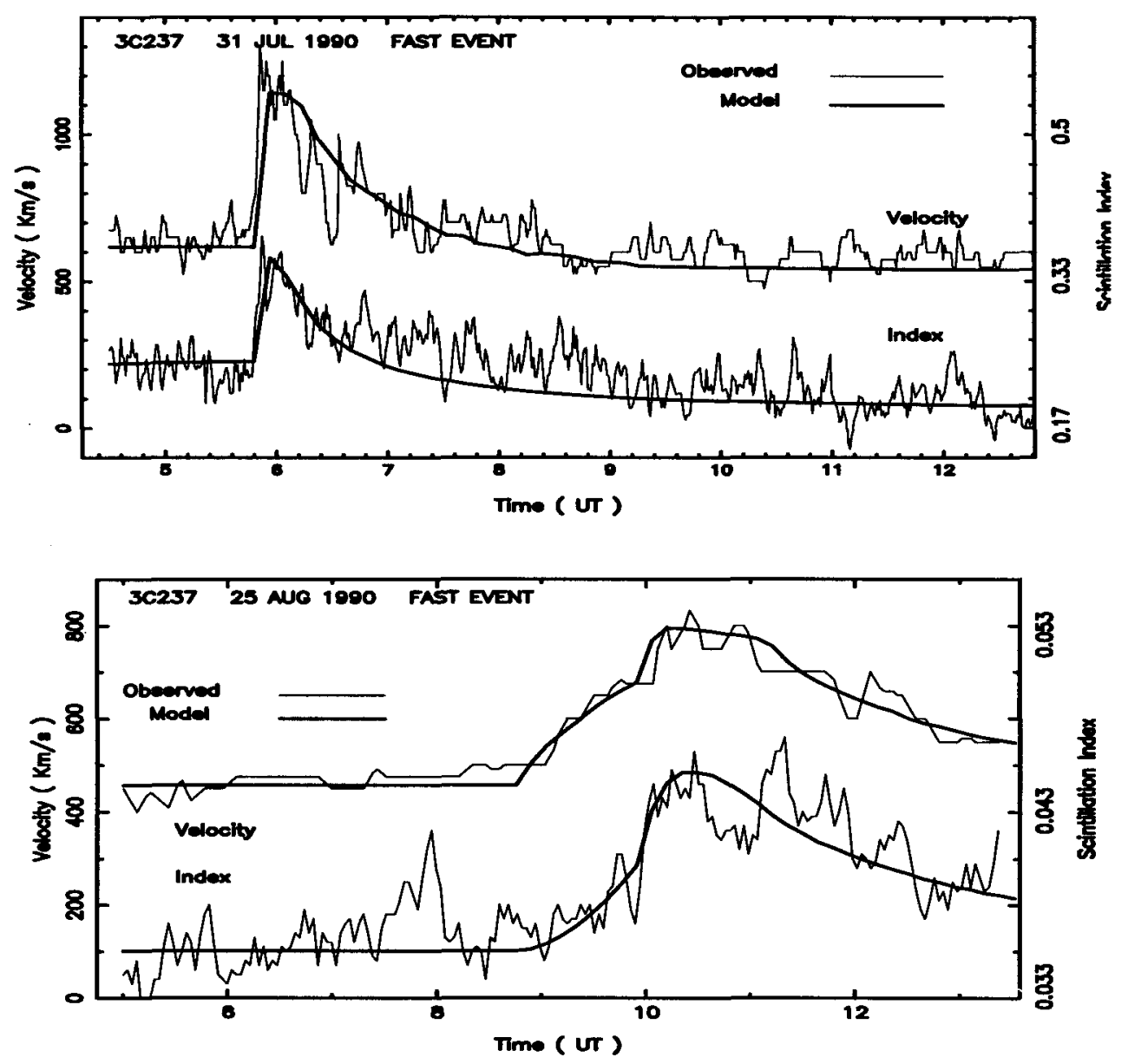

Fig. 1. Interplanetary disturbance of July $31^{\text {st }}$, 1990, was due to Coronal mass ejection at $45^{\circ}$ to the Sun-Earth line on July $30^{\text {th }}$. Extended bow shock associated with the ejecta resulted in sudden ionospheric commencement and rise in $K_{p}$ index on 1 August. On August 24th, 1990, wide spread flare activity was observed directed towards earth. IPS event (lower box) was observed on August $25^{\text {th }}$ which resulted in geomagnetic activity on August $26^{\text {th }}$. 
frequencies. This suggests that the rapid variations in index and velocity, such as observed during the present study, are best detected at smaller elongations and at higher radio frequencies.

Onset-time of the IPS activity and the velocity of interplanetary disturbance was used to study association of IPS events to solar-geomagnetic activity. Each IPS event was mapped to a possible position and time-oforigin at the sun. Simillarly, the event was projected at $1 \mathrm{AU}$ to an expected time-of-arrival of the disturbance at the earth. We considered a window of 5 hours around the expected time of origin and time of arrival while associating the IPS event. Correlation of the time of origin with various solar activities shows that $80 \%$ of IPS events were associated with reports of Coronal Mass Ejections (CMEs) and Long Duration X-ray Events (LDXE). The average mass and energy of the IPS events was found to be $5.3 \times 10^{16} \mathrm{gm}$ and 3.5 $\times 10^{34}$ ergs, respectively, which is in agreement with the study of CMEs and the distribution of their mass and energy (Wagner 1984, Kahler 1992, Jackson 1993). The average opening angle and width of the disturbances was found to $42^{\circ}$ and $8 \times 10^{6} \mathrm{~km}$.

Correlation of the time of arrival of IPS events with the geomagnetic activity implies $50 \%$ of the IPS event resulted in geomagnetic activity in the form of sudden ionospheric commencement followed by a rise in $K_{p}$ index. For these events the IMP- 8 satellite data also showed rise in velocity and density of plasma. We thus suggest that the short-time scale IPS events are the interplanetary signatures of high-velocity CMEs. This is in agreement with current understanding of role of CMEs in solar-terrestrial activity (Webb 1996, Low 1996).

\section{References}

Gapper, G.R., Hewish, A., Purvis, A. \& Duffett-Smith, P.J.:1982, Nature, 296, 633.

Jackson, B.V. \& Howard, R.A.:1993, Solar.Phys., 148, 359.

Kahler, S.W.:1992, Ann.Rev.Astron.Astrophys., 30, 113.

Low, B.C.:1996, ASP Conf. Solar Drivers of Interplanetary and Terrestrial Disturbances, 95, 148.

Manoharan, P.K. \& Ananthakrishnan, S.:1990, MNRAS, 244, 691.

Neugebauer, M.:1987, Conf. Proc. Solar Wind VI, 1, 243.

Tappin, S.J., Hewish, A. \& Gapper, G.R.:1983, Planet. Space Sci., 31, 1171.

Wagner, J.W.:1984, Ann.Rev.Astron.Astrophys., 22, 267.

Webb, D.F.:1996, ASP Conf. Solar Drivers of Interplanetary and Terrestrial Disturbances, 95, 219. 